Michał Jach

\title{
Bericht über die gesamtpolnische Wissenschaftskonferenz: "W służbie społeczeństwu. Polska w obronie praw człowieka na świecie i w kraju”
}

(Im Dienst an der Gesellschaft. Polen bei der Verteidigung der Menschenrechte auf der Welt und im eigenen Land), Krakau, 29. November 2019

Derzeit werden in vielen Regionen der Welt die grundlegenden Menschenrechte immer noch nicht respektiert. Deshalb ist es sehr zu schätzen, dass die Organisatoren für den 29. November in Krakau an der Straße Zyblikiewicza 1, im Club der 6. Luftschutzbrigade, die Konferenz "W służbie społeczeństwu. Polska w obronie praw człowieka na świecie i w kraju” (Im Dienst an der Gesellschaft. Polen bei der Verteidigung der Menschenrechte auf der Welt und im eigenen Land) vorbereitet haben. Federführend war die Päpstliche Universität Johannes Paul II. in Krakau in der Zusammenarbeit mit der Woiwodschaft Małopolska (Kleinpolen), der Polnischen Post und dem militärischen Woiwodschaftsstab in Krakau. Die Schirmherrschaft übernahmen die Kommission des Senats für die Landesverteidigung, der Vorsitzende der Kommission für die Landesverteidigung des Sejms und die Woiwodschaft Małopolska. Die mediale Begleitung erfolgte durch das TVP in Krakau, Radio Krakau, KAI und Gość Niedzielny (Sonntagsgast). Teilnehmer der Konferenz waren an der Thematik Interessierte, sowie Studenten und Soldaten.

Auf der einen Seite sind wir uns bewusst, dass jedem Menschen Rechte zustehen, die aus seiner Menschenwürde hervorgehen: allgemeine Rechte, da sie jeden Menschen betreffen, angeborene Rechte, da sie allein mit dem Fakt des 
Menschseins verbunden sind, unantastbare Rechte, da sie ihm unabhängig von dem System, in welchem der Mensch lebt, nicht genommen werden können. Sie haben auch eine unverzichtbare Charakteristik, denn sie gehören jedem Menschen und niemand kann ihnen entsagen, eine natürliche Charakteristik, da sie bedingungslos existieren, egal ob die gegebene Staatsmacht sie annimmt oder verweigert, und alle zusammen bilden eine sichere Ganzheit. Daher kann keines von ihnen von Irgendjemand entfernt werden. Dennoch muss man die Tatsache feststellen, dass es gegenwärtig Orte in der Welt gibt, an denen dem Menschen sowohl seine persönlichen als auch seine sozialen, ökonomischen, politischen und kulturellen Rechte abgesprochen werden. Deshalb nehmen viele Nichtregierungsorganisationen, aber auch viele Staaten, darunter die Republik Polen, friedliche Bemühungen auf, um unrecht behandelte, verlorene und eingeschüchterte Menschen zu verteidigen. Insbesondere geht es um die Menschen, deren Rechte am häufigsten verletzt werden: Frauen, Kinder, ältere Menschen, Menschen mit Behinderungen sowie um ethnische, religiöse, nationale Minderheiten und Flüchtlinge.

Die Organisatoren der Konferenz hatten sich darum bemüht, die Aspekte der Thematik umfassend zu berücksichtigen und viele bedeutende Vertreter unterschiedlichster akademischer Zentren und Organisationen einzuladen. Unter den geladenen Gästen waren der Metropolit Krakaus, Erzbischof Marek Jędraszewski; der Vorsitzende der Kommission für die Landesverteidigung des Sejms, Michał Jach; der oberste Verwaltungschef Kleinpolens, Piotr Ćwik; der Vizepräses der Polnischen Post, Andrzej Bodziony, ebenso wie Abgesandte folgender Universitäten: Universität Kapstadt, Seattle University School of Law, Päpstliche Universität Johannes Paul II. in Krakau (UPJPII), Jagiellonen-Universität Krakau (UJ), Universität Breslau (UWr), Schlesische Universität, Staatliche Berufshochschule Tarnòw, Technische Militärakademie in Warschau und Medizinisches Militärinstitut in Warschau. Daneben waren diese Institutionen vertreten: Organizacja Narodów Zjednoczonych (Organisation für die Völkervereinigung), Gas Storage Poland, die Polnische Post sowie 4 regionale Logistikstützpunkte.

Die Eröffnung der Konferenz übernahm der Metropolit Krakaus und Großkanzler der UPJPII, Erzbischof Marek Jędraszewski, indem er an den 189. Jahrestag des Novemberaufstands anknüpfte und daran aufzeigte, was es bedeutet, im Dienst des Friedens zu sein, und was es heißt, den Frieden in einem Ausmaß zu festigen, welches die Grenzen unseres Vaterlands überschreitet. Anschließend wurde der Gratulationsbrief des Ministers für Landesverteidigung, 
Mariusz Błaszczak, verlesen. Darin richtete sich der Minister an die Teilnehmer der Konferenz mit folgenden Worten: "Er wünsche effektive Beratungen und interessante Schlussfolgerungen, die im täglichen Dienst an der Gesellschaft angewendet werden können". Dagegen verwies der oberste Verwaltungschef Kleinpolens, Piotr Ćwik, auf die Bedeutung und den Rang der in der Konferenz aufgegriffenen Thematik, indem er die Zusammenarbeit der zahlreichen Zentren und Organisationen unterstrich, die gemeinsam eine lokale Umgebung schaffen, Polen und Europa. Der Vizepräses der Polnischen Post, Andrzej Bodziony, zeigte die vielseitigen Formen auf, wie sich die Polnische Post am Aufbau von Frieden und Menschenrechten im 21. Jahrhundert engagiert und aktiv beteiligt.

Im inhaltlichen Teil der Konferenz griff Dr. Veronica Fynn Bruey (Universität Kapstadt, Seattle University School of Law) in ihrem einführenden Referat Deadly Voyage - the law of displaced people (Tödliche Reise - die Rechte von Geflüchteten) die Frage nach den häufig tödlich verlaufenden Fluchtbewegungen von Migranten auf, deren Ziel Europa ist. Sie erläuterte, dass nicht nur Kriege, ökologische Krisen oder Verstoß gegen die Menschenrechte sondern ebenso ein eklatantes Ungleichgewicht im wirtschaftlichen Bereich zur Auswanderung beitragen. Denn wegen der aktuellen Globalisierung und des immer größeren Informationszugangs durch die technischen Medien fassen viele arbeitsfähige junge Menschen den Entschluss, sich in Richtung ihres vermeintlichen Traumkontinents Europa aufzumachen, um dort ein besseres Leben zu führen. Die Herkunftsländer halten die Fluchtwilligen, deren Rechte in ihren Lebensregionen verletzt werden, häufig nicht auf. Deshalb können sie die Grenzen sogar ohne die entsprechenden Dokumente überschreiten und sich in dem dortigen Land der Prozedur für eine Legalisierung des Aufenthalts unterziehen. Für viele von ihnen kann jedoch dieses Unternehmen zu einer Reise in den Tod werden. Im ersten Teil der Vorträge wurde auch der Themenkreis um das Engagement der Polnischen Post für soziale Sicherheit aufgegriffen (Andrzej Bodziony). Im weiteren Verlauf ging es um die Weltjugendtage, die in religiöser Hinsicht ein Treffen darstellen, das den Jugendlichen Hoffnung und Frieden bringt (H.H. Prof. Dr. habil. Józef Stala), die aber ebenso den Aspekt allseitiger Sicherheit beinhalten (Oberst Adam Jangrot). Der zweite Teil befasste sich mit dem Aufbau einer Axiologie für den polnischen Soldaten (Prof. Elżbieta Osewska), mit der Unterstützung von Militärangehörigen und deren Familien, die Traumata durchleben (Dr. habil. Alicja Senejko, Prof. UWr; Dr. habil. Ewa Gurba, Prof. UJ), wie auch mit der Frage nach dem Dienst der polnischen Militärgeistlichen, die überall anwesend sind, wo polnische Soldaten eingesetzt 
werden (H.H. Oberst SG Dr. Zbigniew Kępa). Für den anschließenden Teil waren drei parallele Workshops organisiert: 1. Wojsko Polskie w świecie - misja i powrót $z$ misji i co dalej (Polnisches Militär in der Welt - die Mission, die Rückkehr aus der Mission und was danach kommt), Leiter: Oberst Adam Jangrot, Dr. Magdalena Butrymowicz; Referenten: H.H. Oberst Piotr Kowalczyk, Dr. Bartosz Kucia, Dr. Magdalena Butrymowicz, Melanie Clerk; 2. Instytucje prywatne $w$ służbie praw człowieka - wzajemna wspótpraca $i$ koordynacja obronności Polski (Private Institutionen im Dienst an den Menschenrechten - die gegenseitige Zusammenarbeit und Koordination der Verteidigungsinitiativen Polens), Leiter: Dr. Michał Leski; Referenten: Beata Wittmann, Lech Skowron; 3. Problemy rodzin żolnierzy - praca socjalna z rodzina żolnierzy (Probleme der Soldatenfamilien - Sozialarbeit mit der Soldatenfamilie), Leiter: Dr. habil. Małgorzata Duda, Prof. UPJPII; Referenten: Dr. Barbara Sordyl-Lipnicka, Dr. habil. Alicja Senejko, Prof. UWr., Dr. habil. Małgorzata Sobol-Kwapińska, Prof. UWr.

Ein herzlicher Glückwunsch gebührt den Organisatoren, vor allem von der Päpstlichen Universität Johannes Paul II. in Krakau - H.H. Prof. Dr. habil. Józef Stala und Dr. Magdalena Butrymowicz - die diese Konferenz grundlegend inspiriert, aber ebenso souverän geleitet und vortrefflich zusammengefasst haben. Es ist sehr gut, dass derartige Themenkreise und Fragen rund um das Engagement Polens bei der Verteidigung der Menschenrechte auf der Welt und im eigenen Land aufgegriffen wurden und derartig mannigfaltig und aspektenreich Gewinn brachten. Deshalb bin ich überaus zufrieden, dass die Errungenschaften dieser Konferenz in der Monographie vieler Autoren präsentiert werden, die in dem landesweit und über die Grenzen hinaus bereits gut bekannten und geachteten Wissenschaftsverlag der Päpstlichen Universität Johannes Paul II. in Krakau erscheint. 